\title{
What Happens inside Pulsars?
}

IT is now generally accepted that pulsars are associated with rotating neutron stars, but there is no really satisfactory detailed explanation of the mechanisms whereby the rotational energy of the star is transformed into the kind of radiation observed. NP 0532 in the Crab Nebula is the youngest pulsar known; it is still emitting visible and $\mathrm{X}$-ray pulses as well as radio pulses and has been the object with the greatest interest to observational astronomers-and often a frustrating source of negative information which pulls the rug from under almost any detailed model put forward by the theoreticians. There was an outstanding illustration of this behaviour in September last year, when there was a sudden increase of this pulsar frequency. Having confounded much of the astronomical community by this change, NP 0532 perversely refused to suffer other similar events, even though the "starquake" model put forward to account for them required that they should occur at least once a month.

New insight into the mechanisms operating in this pulsar (and presumably in others too) is provided by the work reported on page 445 of this issue of Nature. Observations of the optical pulses from NP 0532 were made at the Smithsonian Astrophysical Observatory for the period September 1969 to April 1970, which includes the occasion of the marked jump in frequency. The measurements are sufficiently accurate to point to the dominant mechanism in the transfer of rotational energy into radiative energy. This accuracy is obtained by studying the pulsar over several consecutive nights. Extrapolation from the end of one night's observations to the beginning of the next is effected by a least squares fit to the pulsar's previous behaviour. The sudden jump in frequency on September 22, 1969, seems to have caused a cumulative deviation from the predicted behaviour on subsequent nights, and because this deviation is non-linear at least one other parameter than the frequency of the pulsar must have changed. There is a great deal of evidence for disturbed activity after the jump, but the overall behaviour is close to that expected of a system in which magnetic dipole radiation is the dominant mechanism.

Even better agreement with the predictions of this model is obtained when the behaviour of the pulsar just before the jump is interpreted rather differently than has been accepted previously. The original report on the frequency change from the Arecibo radio observatory described the erratic behaviour of the pulsar just before the jump as "quasi-sinusoidal", and this has been taken in some quarters as evidence for a planetary companion to NP 0532. Unfortunately, like so many experimental data, there is no unique curve fitting the observations, and in this case the changes in the data can be well fitted by two straight lines rather than one sine curve. This fitting now seems much more plausible, for, in conjunction with the magnetic dipole radiation law and assuming also a small change in the moment of the dipole (only about 1 part in 2 million), there is good agreement between the theory and subsequent observations.

Small errors forming a background noise are present throughout the data; although even the detection of this very low level noise would have seemed a triumph a few years ago, it is sufficient to prevent any possibility of using the variations in pulsar frequency caused by the Earth's movement in the Sun's gravitational field to measure accurately the gravitational redshift. In spite of this extra blow to the hopes of some theoreticians (which emphasizes the apparently capricious nature of pulsars), there may be some comfort in the suggestion that there really are starquakes on NP 0532, and that it is the frequent occurrence of rather small quakes which produces the noise.

\section{GALAXY FORMATION Early Protospirals}

\section{from our Observatories Correspondent}

JAN OoRT, who retired this year as director of the Leiden Observatory, has made important contributions to the study of the structure of our Galaxy. Recently one of his principal concerns has been the origin of the high velocity clouds of neutral hydrogen which are observed to be falling into the galactic plane at high and intermediate galactic latitudes. The clouds, which have velocities of up to about $200 \mathrm{~km} \mathrm{~s}^{-1}$, have been detected by $21 \mathrm{~cm}$ observations made mostly at the Dwingeloo radio observatory in Holland. In the past Oort and his collaborators have discussed two possible origins for the clouds. One explanation is that they result from giant explosions in nearby spiral arms which have ejected neutral hydrogen into the galactic halo. According to this view it is largely by chance that the material is observed to be falling into the galactic plane in the vicinity of the Sun. In any case Oort has rejected this explanation (Astronomy and Astrophysics, 7 , 381; 1970), and instead has concluded that intergalactic gas is streaming into the Galaxy. A rough estimate of the total flow is $2 \times 10^{17}$ hydrogen atoms per $\mathrm{cm}^{2}$ of the galactic plane per million years. This corresponds to an increase of the Galaxy's mass of 0.9 per cent in $10^{9}$ years.

Oort attempts to relate the observation of infalling hydrogen to a theory of the formation of galaxies. $\mathrm{He}$ first considers the origin of the angular momentum of spiral galaxies. This subject has had a controversial history. Many theoreticians suppose that galaxies formed as a result of gravitational instability soon after the decoupling of matter and radiation in the Universe. At that time the scale of the Universe was 1/1,000 of its present value. Spiral galaxies could not have contained their present angular momentum because they were too small. Starting with Hoyle in 1951, various people have explored the possibility that angular momentum was imparted to protogalaxies by extraneous couples-for example, a nearby cluster of galaxies. 